\title{
The bio-genetics of male homosexuality and the contemporary global societal discourse
}

\begin{abstract}
The global discourse stated in this article will remain topical in perpetuity, in the author's view, though the text and dynamics of the discourse would keep changing as the global society evolve, and as new scientific evidence emerge. However, even the advent of new scientific evidence does not mean that the societal views would change immediately or automatically, as Galileo Galilei, the central figure in the transition from natural philosophy to modern science and in the transformation of the Scientific Renaissance into a scientific revolution, bitterly found out during his lifetime.

Then enter the increasingly vociferous Lesbian Gay Bisexual and Transgender (LGBT)'s strong society and its dedicated and ardent campaigners buoyed by the ever increasing scientific evidence that appear to support their sexual orientation and views.

The battle lines have been drawn between the smaller but global LGBT and a larger religious, cultural and traditional global opponent. More researches are still ongoing and the outcomes would lead to more revelations. Some of the LGBT opposition in the West appears to be weakening by the evolving society and the scientific evidence. This is evidenced in the recent political and legal exploits for the LBGT society in the West. For its large and strong opponent in the so called Third World society, the story is very different. However, this article has mainly dealt with male homosexuality.
\end{abstract}

Volume 9 Issue 4 - 2018

Anthony Emeka Madu

Obstetrics and Gynaecology, RCOG/FSRH/I LM, UK

Correspondence: Anthony Emeka Madu, Specialist Registrar, Obstetrics and Gynaecology, RCOG/FSRH/I LM, UK, Tel +44-793-I62-63I5, Email emymadu@yahoo.co.uk

Received: June 06, 2018 | Published: July 18, 2018

\section{Introduction}

The biology of male homosexuality may have been there for centuries as humans evolved, after all, the hypothalamus, the centre in the brain that controls sexual behaviour, has been there as humans evolved. Born this way, some would argue. However, our understanding of the biology of homosexuality, appear to be in its evolutionary infancy. As new evidence from research emerges, the fascination of this important feature of humanity grows.

\section{Global discourse}

There are very strong contrasting religious, cultural and wider societal narratives that are still growing in many regions of the world. For the mainstream Christian societies, homosexuality is sin. They cite verses in the Bible to consolidate their strong views. Some from developing countries identify the homosexuality matter as a Western phenomenon; that humanity is imperfect; heterosexuality is not perfect and that all need conform to norms of nature. They argue that reproducing naturally if biologically fertile is that homosexuals were conceived and born mostly through natural means as offspring of their parents (a man and woman) naturally, or through are Artificial Reproduction Techniques (ART) by couples who were unfortunate not to conceive naturally. They say if you are a natural product of a man and woman, it is only natural you do same if you are fertile and potent, so that the natural cycle of reproduction continues.

Same, many argue, applies to co-habitation as man and woman or husband and wife. Some have asked why someone who does not like or say he was not made to like a woman's body or part of it, would then like to have and keep offsprings that of a woman's body or part of it. Heterosexuality they say does not confer $100 \%$ perfection in sexual orientation in whatever definition you use. These people argue if everyone is homosexual, humanity could be extinct but for ART. The same logic is used by these people to advance their argument that if other mammalian species were to adopt same homosexual orientation, they would be extinct unless humans intervene with use of ART to keep the mammalian species from extinction. Regardless of how these exaggerations of the global discourse has been or may become, some feel that human beings are acting as if they alone own the planet and want to destroy nature and climate. This line of thought would surely unnerve homosexuality campaigners and sure to spur counter arguments, as the societal debate continues to evolve.

ln some countries, you may be stoned to death if you practice homosexuality and in some, there are strong legislations criminalizing homosexuality. In Nigeria, for instance, the author understands that you can be jailed for up to 14 years if you practice homosexuality, and ratification of this legislation appeared to have been ratified only in 2014. Nigeria is not alone in this; The Gambia, Uganda, Kenya and other countries around the world have formal adversarial antihomosexual legislations which clearly reflect the mood and stance of their respective societies.

In Bermuda, a British Overseas Territory, a court last year legalized same sex marriage' However, the ledgislature' intervened to overturn this legalization, declaring that marriage should be define as union of a man and woman, in line with the mood and opinion of its society.

The union of man and woman as husband and wife, many say, is natural. However, some argue that naturalness was a cognitive bias. Some who disagree with the latter argue that it was intellectual laziness not to know what naturalness or normality meant or stood for.

Some same sex homosexual celebrities who recently posted ultrasound pictures of expectant babies from surrogate mothers received strong objections from some section of the public who argue that this amount to renting a woman's womb.

However, in the West, there are increasingly more favourable legislations and an increasingly more tolerant society but not among 
the section of the societies with strong religious persuasion' Also gaining more recognition and acceptance are the phenomenon of bisexuality and transgenderism. However, these are also confronted with strong opposing views from the section of the western society with strong religious and traditional persuasions. Most of these successes in gaining acceptance in western societies appear to be driven by the tireless efforts of dedicated campaigners, buoyed by powerful and rich financiers.

Subsequent paragraphs in this paper give insight to the ever increasing scientific evidence that homosexual men were born that way.

\section{The bio-genetics}

In the past, some studies of sexuality had focused on men for decades, yet there is evidence that sexual orientation is partly inheritable in the male. Consider the mid 1990s: The findings were that genetic variations exist in the regions of the $\mathrm{X}$ chromosome and chromosome 8 , that were linked to homosexuality. At the time, no specific genes were identified. Additionally, there was no explanation as to why men are likely to be homosexual if they have older brothers, the so called "fraternal birth order effect."

Dean Hamer was credited to having being the first to identify a genetic region linked to homosexuality, in 1993.

In 1991, Simon LeVay, a neuroscientist discovered that the size of hypothalamus in heterosexual men differs from that of homosexual men, which is smaller. However, it has been known for longer periods that this region is responsible for regulating much behaviour including sexual behaviour in mammalian species.

From current researches and the ones that are ongoing, it appears that the scientific community is getting closer and closer to understanding the origins of homosexuality. Current studies suggest that science is getting close to identifying the pathways that shape sexual orientation in men, ${ }^{1}$ and that genes and antibodies are involved in shaping this complex pathway.

Two genes that may play a part in sexual orientation had been identified, and there appear to be an explanation about the fraternal birth order effect.

In a study comparing DNA from 1231 heterosexual and 1077 homosexual men, ${ }^{1}$ researchers identified two variant genes, and one is linked to chromosome 13 and is called SLITRK6. The latter is stated to be active in the hypothalamus of male mice fetuses before they are born. This clearly suggests this could be the crucial time when sexual differentiation is occurring.

The second gene called TSHR is on chromosome 14 and helps regulate thyroid function. Evidence from this comes from the fact that TSHR is disrupted in Grave's disease, a genetic disorder common in homosexual men. Not all men with these genetic variant would have homosexual behaviour, as many other factors including the environment are considered to have influence of homosexuality.

Some have suggested that multiple genes may be involved, with each of these having their contributory small effect.

\section{Maternal contribution}

Some researchers have suggested that maternal immune system play a part in sexual orientation. It is a known fact that our body's immune system recognizes particles from infectious or harmful agents that come into contact with the body. The pregnant state is a different immunological state compared to the non-pregnant state. 1t is a fact that sometimes the pregnant woman can produce antibodies against fetal particles like the blood group (for instance; rhesus incompatibility). There is now evidence that maternal antibodies against fetal brain protein molecules (made only in males) may be involved in the fraternal birth order effect. ${ }^{2}$

The protein molecule, researchers had concluded, was good as it is produced from surface of the brain, making it easy for antibodies to find or detect it. The protein is also a good candidate as it affects how neurons communicate with each other. In this study ${ }^{2}$ of 142 women screened for antibodies to a particular brain protein, researchers found the following in the decreasing order of the level of antibodies against this protein:

a. Mothers of homosexual males with older brothers.

b. Mothers of homosexual males with no older brothers.

c. Mothers who had heterosexual sons.

\section{d. Women with no sons at all.}

From this study, the researchers were of the view that these type of antibodies increase in these women with every male baby they become pregnant with, as we observe in rhesus incompatibility. At certain levels of antibody concentration yet to be clarified, it is possible for the effect of these antibodies on the targeted brain proteins cause some changes in brain development in-utero, and this in turn influences sexual orientation.

Though the study population in this research is small and its findings needed to be clarified by larger studies, its findings could lead the way to understanding of the origins and development of male homosexuality.

\section{Some subtle divergence to spur further curiosity}

Some animal studies showed the cells that control sexual behaviour in female mice (those that have not mated and those that are new mothers), are located at the edge of hypothalamus in contrast to cells for aggression which are located in the centre. For the male mice, the cells for these two behaviours are mixed and overlap. ${ }^{3}$

The hypothalamus look similar across species and the difference in male and female does not mean different brain. This is because other regions known to look different in the two sexes in the mouse brain have much overlap in the brain of human.

The author is neither suggesting the findings of this study apply to human, nor comparing human and animal sexual behaviour to homosexuality. Sexual behaviour, is understandably much more complex and context-dependent in humans compared to lower mammalian species. However, the author speculates that the biology and genetics of female homosexuality could well be different from that of the male homosexuality, as new evidence emerges from ongoing studies.

Human scientific curiosity would continue to increase and advance in perpetuity, in its ever increasing quest to find out more information. Recently, there have been reported advances in Prenatal Testing. Researchers isolated many types of fetal cells from maternal blood and sequencing those that looked more intact. This is a more painstaking 
variant of the already existing Prenatal Testing Technique and capable of more information on the unborn baby. This new test could one day be in routing use. From the whole-genome sequencing in-utero, ${ }^{4}$ we know that genetics could inform prospective parents of choices relating to conception. This could even digress from having designer babies usually modeled to avoid severe genetic anomaly, to designer babies modeled to avoid subtle variation in genetic information. Identifying subtle genetic information can take prospective parents into new grounds. Some advocacy groups have long suggested that identification of some genetic variations with adverse societal implication could increase discrimination.

Advances in assisted reproduction techniques usually come with controversy. Questions or concerns of ethics, policy, safety and efficacy appear once a technology becomes public, and new suggestions on how this could be used usually sets off a cascade of debate. One clear example the use of the Mitochondrial Replacement Therapy (MRT) in the UK. The latter involves creating a baby from three people to prevent the passing of severe mitochondrial disease;

A. Nucleus of a woman's egg.

B. Mitochondria of another woman's egg.

C. A sperm.

However, the author is aware that the debate has now turned to focus on another specific use of MRT; that is allowing same sex female couples to have a genetically related offspring.

Currently, there is no means to share genes between two eggs. The genetic relatedness between the mitochondrial donor and the offspring is only about $0.2 \%$. To those who suggest using MRT in this way, this degree of genetic relatedness seems a way of fulfilling the need for genetic relatedness. They argue that MRT falls within what is described as reproductive freedom for heterosexual couples and wondered by same-sex couples should not have same freedom. There is no denying that the desire for genetic relationship is a strong one irrespective of gender or sexuality, however, it would be difficult to convince many why MRT designed to prevent severe debilitating inheritable mitochondrial disease, should be used in another way; that is to have $0.2 \%$ genetic relatedness for same-sex couples, which appear to be comparatively, a less compelling reason. This shows how far this sort of global debates can go.

The author suggests that there is a risk that now or in the future, parental choices could be affected by subtle genetic information. This, the author speculates and fears, could one day advance to parents wishing to choose sexual orientation and behaviour of the unborn fetus, raising more ethical questions.

Thus, as science advances further, the global discourse and philosophical realms will do the same, forcing some to conclude that humanity's quest to find ways to undermine nature could finally destroy humanity.

\section{References}

1. Sanders AR, Beecham, GW, Guo S, et al. Genome-wide association study of male sexual orientation. Scientific Reports. 2017;7:1-6.

\section{PNAS.}

3. Hashikawa K, Hashikawa Y, Tremblay R, et al. Esr1+ cells in the ventromedial hypothalamus control female aggression. Nature Neuroscience. 2017;20:1580-1590.

4. Perinatal Diagnosis. 